\title{
Response of the groove in heliospheric Lyman- $\alpha$ glow to latitude-dependent ionization rate
}

\author{
M. Bzowski ${ }^{\star}$ \\ Space Research Centre PAS, Bartycka 18A, 00-716 Warsaw, Poland \\ Received 25 February 2003 / Accepted 16 June 2003

\begin{abstract}
I study the model response of the latitudinal profile of the heliospheric Lyman- $\alpha$ glow to various profiles of the ionization rate. The two profiles are characterized by their range separately in the north and south hemisphere, and by the contrast between the equator values and north and south pole values. These parameters define a mesh of the input ionization rate profiles, used to derive the output heliospheric glow profiles. It is shown that in the crosswind plane containing the Sun there exists a linear correlation between the north and south ranges of the ionization profile and the corresponding ranges of the glow profiles. This correlation does not depend on the contrast of the ionization profile. The contrast of the glow profile is almost linearly correlated with the contrast of the ionization profile, but this correlation changes with the change of the input profile width. Based on these correlations, a method to derive the latitudinal profile of the solar ionization rate from observations of the heliospheric glow at $1 \mathrm{AU}$ is proposed.
\end{abstract}

Key words. interplanetary medium - solar wind - Sun: UV radiation - Ultraviolet: solar system

\section{Introduction}

The aim of this paper is to present models used to derive parameters of the ionization field of heliospheric hydrogen from remote-sensing observations performed by the SWAN experiment onboard SOHO and presented by Bzowski et al. (2003, this issue, Paper II). I present a brief resume of the history of the research on solar wind asymmetry and its remote sensing by observations of the heliospheric Lyman- $\alpha$ glow.

The paper presents results of model calculations of profiles of the heliospheric backscatter radiation intensity as a function of heliographic latitude for various profiles of the ionization rate.

\section{Modeling}

The idea of modeling presented in this paper is to take a latitudinal profile of the ionization rate similar to that observed by Ulysses during the 1995 fast latitude scan (Phillips et al. 1995; Bzowski 2001a, see Fig. 1) and to calculate the latitudinal profile of the Lyman- $\alpha$ glow. Ulysses observed a flat but noisy enhancement of the solar wind mass flux (the "bulge"), straddling almost symmetrically the solar equator. Accordingly, I adopted input ionization rate profiles featuring a flat, symmetrical enhancement in the equatorial region. From the calculation of a grid of such models, where the variable parameters were the width and height of the input ionization profile, I obtained an output grid of glow profiles which feature broad minima in equatorial band of latitudes (the "groove"). With these profiles on hand, I studied correlation between the ionization and glow

\footnotetext{
* e-mail: bzowski@cbk.waw.pl
}

profiles. In particular, I was looking for the correlation between the north and south latitudinal range of the bulge on one hand and the latitudinal range of the groove on the other hand, and for the correlation between the depth of the groove and the height of the bulge.

The approach for modeling of the intensities of heliospheric glow was essentially the same as in the paper by Bzowski et al. (2002). The intensities were calculated using the singlescattering, optically thin model:

$I\left(r_{0}, \theta, \delta\right)=I_{\odot} \frac{r_{\mathrm{E}}^{2}}{4 \pi} \sigma n_{0} \int_{r_{0}}^{\infty} \tilde{n}(r, \theta, \delta) / r^{2} \mathrm{~d} r$.

In this formula, $I\left(r_{0}, \theta, \delta\right)$ is the intensity of the backscattered radiation observed along a heliocentric line of sight anchored at the distance $r_{0}$ from the Sun in a point whose radius vector $\boldsymbol{r}_{0}$ in a heliocentric reference system makes angle $\theta$ with the upwind direction and $\delta$ with the solar equator (that is, $\delta$ is its heliographic latitude); $I_{\odot}$ is the solar illuminating flux, assumed to be spherically symmetric; $r_{\mathrm{E}}$ is $1 \mathrm{AU}$ ( $r_{0}=r_{\mathrm{E}}$ throughout); $\sigma$ is the cross section for interaction of solar Lyman- $\alpha$ photons with neutral hydrogen atoms, averaged over the solar Lyman- $\alpha$ line width; $n_{0}$ is the gas density at the termination shock (formally: at infinity); $\tilde{n}(r, \theta, \delta)$ is the local density of the gas, taken as a fraction of $n_{0}: \tilde{n}(r, \theta, \delta)=n(r, \theta, \delta) / n_{0} ; n(r, \theta, \delta)$ is the local gas density in a point defined by radius vector $\boldsymbol{r}(r, \theta, \delta)$. In the calculations to follow, $\theta$ was always equal to $90^{\circ}$ and since the lines of sight were directed radially away from the Sun, the latitude of each point on a given line of sight was equal to one $\delta$ value, specific for this line of sight. As it is evident 
from Eq. (1), the gas density at infinity $n_{0}$ and the solar illumination of the gas $I_{\odot}$ are multiplicative factors that cancel when relative intensities are calculated.

The relative intensities $Q(\delta)$ are defined as:

$Q(\delta)=\frac{I\left(r_{0}, \theta, \delta\right)}{I\left(r_{0}, \theta, \delta_{\min }\right)}-1$

where $\delta_{\min }$ is by definition the heliographic latitude of the line of sight which returns the minimum intensity in the scan plane.

The underlying density of the gas $\tilde{n}(r, \theta, \delta)$ was calculated using the model developed by Bzowski et al. (2002). In this model, which is based on the model by Ruciński \& Bzowski (1995), the density is calculated as a numerical integral of the distribution function over the velocity phase space. The distribution function $f_{\text {LOC }}$ for velocity vector $v$ in a selected location in space, determined by the heliocentric distance $\boldsymbol{r}(r, \theta, \delta)$, is calculated as a product of the assumed distribution function at the termination shock $f_{\infty}$ for a velocity vector $\boldsymbol{v}_{\infty}$ and of the ionization weight $w_{\text {ion }}$ :

$f_{\mathrm{LOC}}(\boldsymbol{v}, r, \theta, \delta)=f_{\infty}\left(\boldsymbol{v}_{\infty}, r_{\infty}\right) w_{\text {ion }}\left(\boldsymbol{v}, r, \theta, \delta, \boldsymbol{v}_{\infty}, r_{\infty}\right)$.

Here $r_{\infty}$ is the (formally infinite) distance from the Sun, where one must assume some form of the distribution function of the gas $f_{\infty}\left(\boldsymbol{v}_{\infty}, r_{\infty}\right)$. Basically, this function is quite complicated since the hydrogen gas is heavily processed by the heliospheric interface (Izmodenov et al. 2001). In practice, it is assumed that the gas distribution function at $r_{\infty}$ is a Maxwellian with a temperature $T_{\infty}$, travelling with the bulk velocity vector $\boldsymbol{v}_{\mathrm{B}}$ (see also Fahr 1978; Thomas 1978; Wu \& Judge 1979; Lallement et al. 1985; and a review by Ruciński \& Bzowski 1996). The velocity vector $\boldsymbol{v}_{0}$ of the atom at $r_{\infty}$ is calculated numerically by back-tracking the atom from its position $(r, \theta, \delta)$ inside the heliosphere, where it has velocity vector equal to $\boldsymbol{v}$, to $r_{\infty}$. In this particular embodiment of the density model I assumed that the pressure of solar Lyman- $\alpha$ radiation is constant in time, so the atoms were following hyperbolic trajectories.

The ionization weight $w_{\text {ion }}\left(r, \boldsymbol{v}, \theta, \delta, r_{\infty}\right)$ is the probability that the atom survives such a travel in the ionization field, described by the equation

$\beta(r, \delta)=\beta_{0}(\delta)\left(\frac{r_{\mathrm{E}}}{r}\right)^{2}$

where $\beta_{0}(\delta)$ is one of the profiles of the ionization rate at $1 \mathrm{AU}$, presented below. The ionization weight is calculated numerically by the following integration:

$w_{\text {ion }}=\exp \left[-\int_{t_{0}}^{t_{1}} \beta_{0}(\delta(r, t))\left(\frac{r_{\mathrm{E}}}{r}\right)^{2} \mathrm{~d} t\right]$

where $\delta(r, t)$ is the instantaneous heliographic latitude of the test atom during its travel from the local point to $r_{\infty}$. Since the atoms do not follow radial trajectories, it usually changes considerably during the flight of the atom.

The calculations were performed for the bulk velocity and temperature of the hydrogen gas at the nose of the termination shock similar to those derived by Costa et al. (1999): $v_{\mathrm{B}}=20 \mathrm{~km} \mathrm{~s}^{-1}, T_{\infty}=12000 \mathrm{~K}$. The ecliptic coordinates of the

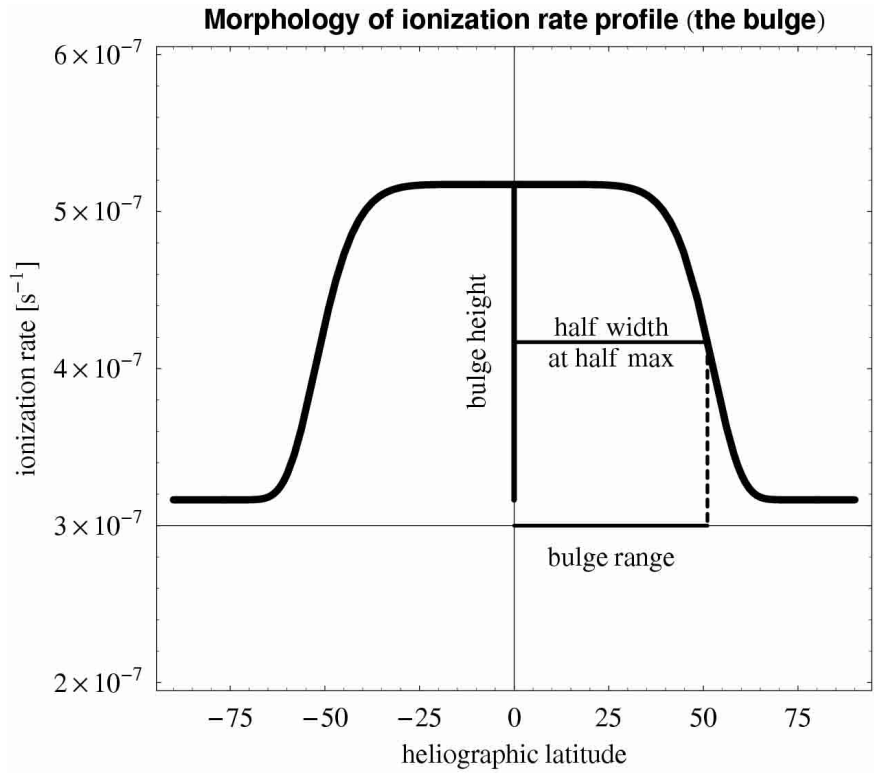

Fig. 1. Definition of morphological features of the ionization bulge profile. The range corresponds to half width at half maximum and the height is the ratio $\beta_{0}(0) / \beta_{0}(90)$. The width of the profile is equal to the range. The range is expressed in degrees of heliolatitude and the height is dimensionless.

upwind direction were assumed as $\lambda_{\mathrm{B}}=252.4^{\circ}$ and $\phi_{\mathrm{B}}=7.5^{\circ}$. The values of the ionization rate were assumed constant in time, and the value of the radiation pressure was adopted as for the time of Ulysses fast latitude scan in 1995: $\mu=1.07$. For the verification modelling, discussed in Paper II, the values of radiation pressure derived from the EUV' 97 model by Tobiska et al. (1997) and presented by Bzowski (2001b) were used.

As input to the modelling, I used a series of helio-latitudinal profiles of the ionization rate that throughout this paper and Paper II will be referred to as the ionization bulge. This is because the model ionization rate at $1 \mathrm{AU}$, when rendered in 3D, appears as a spherical surface with a bulge in a band of equatorial latitudes. I checked extensively one class of models: symmetric about the solar equator, characterized by the two largescale morphological parameters defined in Fig. 1. They are the bulge width, defined precisely as half width at half maximum of the profile, and the bulge height, defined as the ratio of the values at the equator and at the poles. In the modelling, I used as the base value the rate observed at the solar poles by Ulysses during the Fast Latitude Scan in 1995 (Phillips et al. 1995), plus the photoionization rate $3.2 \times 10^{-7} \mathrm{~s}^{-1}$, inferred from the $10.7 \mathrm{~cm}$ proxy (for discussion, see Bzowski 2001b). Since the adopted profile is symmetric with respect to the equator, its north and south widths - by assumption equal to each other - are also equal to its north and south range. This is not the case, however, for profiles not symmetric with respect to the equator, as those presented in Fig. 9, which will be discussed in a further part of the paper.

The modelling was performed on a mesh of 7 widths and 6 heights of the profiles, presented in Fig. 2. For these profiles, I calculated intensities of the heliospheric glow for lines of sight directed away from the Sun from $1 \mathrm{AU}$, located in the 


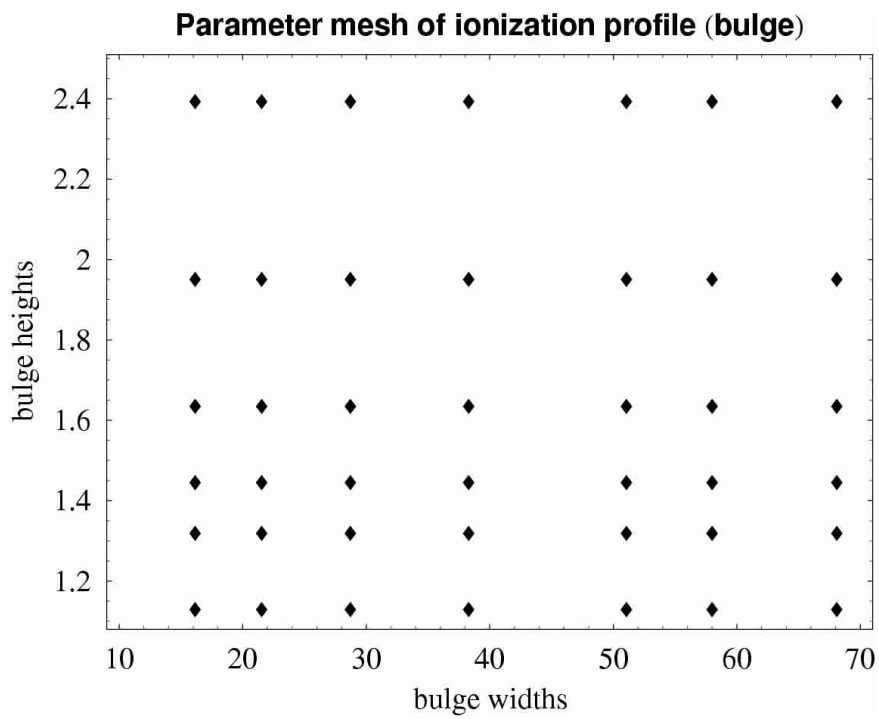

Fig. 2. Mesh of the ionization bulge parameters used in the modelling of the helioglow intensity groove profiles.

plane perpendicular to the gas inflow axis. The sampling was every $7.5^{\circ}$. The scan plane is close to the Ulysses orbit and comes close to but not precisely through the ecliptic and solar poles.

This was the baseline geometry of the input bulge profiles. Since the observed profiles of the groove, presented in Paper II, suggest that the ionization bulge is not always symmetric, I studied qualitatively the following two additional cases: when the bulge is shifted slightly (by $5^{\circ}$ ) and considerably (by $30^{\circ}$ ) to the south. In these two cases, calculated for all 7 widths, but for only one height of the ionization bulge, the north and south ranges of the ionization profile were no longer equivalent to its widths.

From the modelling performed I obtained a family of profiles of the groove whose morphological parameters I define as shown in Fig. 3.

In this geometry, although the solar ionization rate is north-south symmetric, the resulting intensity profile is not. Consequently, I consider separately the north and south profile ranges, widths and depths, as indicated in the figure. I refer the north and south half-width of the profile to the minimum of the groove, marked with the vertical axis in the figure. The minimum does not occur at the heliographic equator. The groove range to the north and to the south in heliographic coordinates are taken with respect to the solar equator while the widths of the profile with respect to the latitude of the minimum.

\section{Modeling results}

In this section, I will discuss first the main features of the groove profiles created by the ionization symmetric about the solar equator. I will show the morphological features important for correct interpretation of the observed profiles of the glow and will point out the quantitative correlations that enable determining the parameters of the ionization profile from observed profiles of the glow. Then I will qualitatively show modifications of the glow profile when the ionization profile

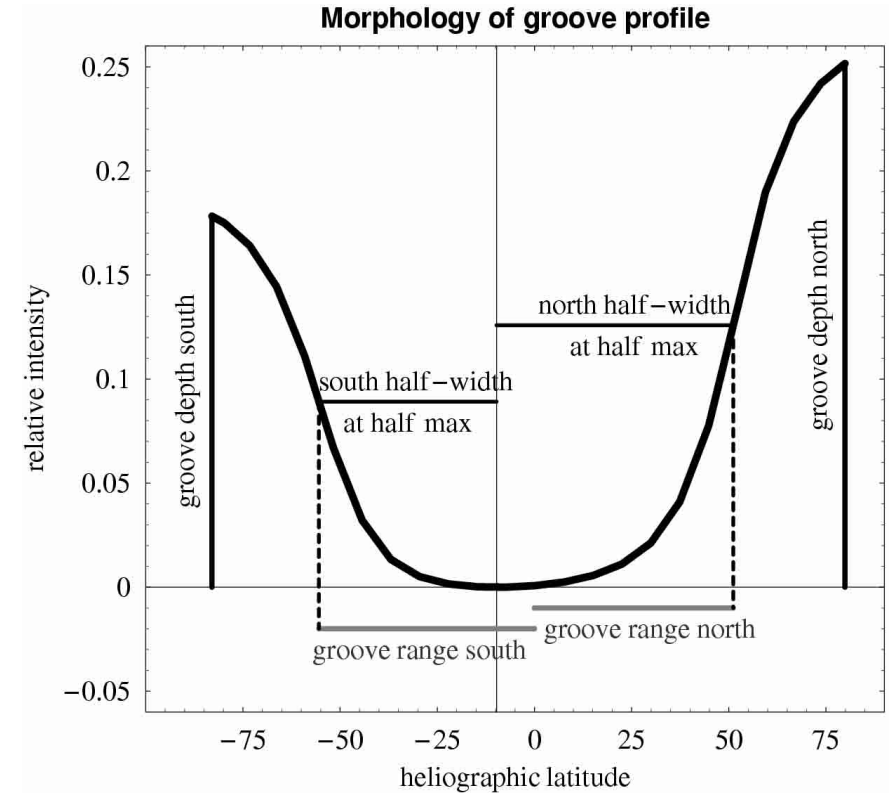

Fig. 3. Morphology of the groove profile with respect to heliographic latitude in the plane perpendicular to the inflow direction. The vertical line marks the minimum of the profile which occurs a few degrees to the south from the equator. The north (south) range of the profile is defined as the latitude of its half-maximum in the northern (southern) hemisphere. The north (south) width of the profile is defined as the difference between the latitude of the north (south) half-maximum and of the latitude of the minimum of the profile. The north and south depths $q_{\mathrm{N}}, q_{\mathrm{S}}$ are the ratios of the intensities at the poles and at the equator (see Eq. (2)). The north-south asymmetry of the profile $q_{\mathrm{N} / \mathrm{S}}$ is defined as the ratio of the north depth to the south depth: $q_{\mathrm{N} / \mathrm{s}}=$ $q_{\mathrm{N}} / q_{\mathrm{S}}$ The widths and ranges are expressed in degrees of heliographic latitude, and the depths and depth asymmetry are dimensionless.

is no longer symmetric with respect to solar equator. Finally, I will define the procedure to determine the parameters of the ionization rate profile from the observed parameters of the glow intensity.

\subsection{Ionization bulge symmetric about the equator}

Example profiles of the groove for different widths of the ionization bulge are presented in Fig. 4. With the increasing range of the bulge, the groove profiles become flatter and flatter both in terms of the poles-to-equator ratios and in terms of the flatness of the bottom of the profile. However, there exists a threshold value of the bulge width, below which the depth of the groove becomes to decrease again: in Fig. 4 the groove is the deepest for the bulge width equal to $\sim 29^{\circ}$.

\subsubsection{North-south asymmetry of the groove}

The north-south asymmetry of the profiles is defined as $q_{\mathrm{N} / \mathrm{S}}=$ $q_{\mathrm{N}} / q_{\mathrm{S}}$, where $q_{\mathrm{N}}, q_{\mathrm{S}}$ are the north and south depths of the groove, defined in Fig. 3. The behavior of $q_{\mathrm{N} / \mathrm{s}}$ for various widths and heights of the ionization bulge is summarized in Fig. 5. The asymmetry is caused by the small inclination of the gas inflow axis to the solar equator. Generally, it attains the 


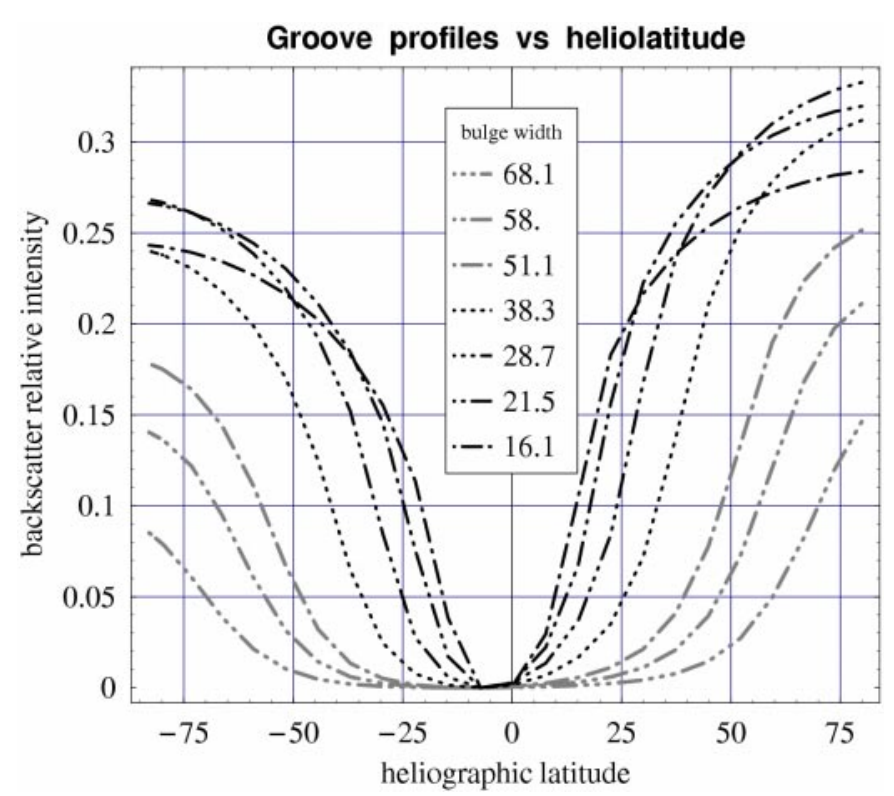

Fig. 4. Family of the (relative) groove profiles as function of heliographic latitude for the widths of the ionization rate indicated in the box and height of the bulge equal to 1.63 .

maximum values for medium widths and tallest heights of the ionization rate profile. The most important here are the upper limits for the N/S asymmetry of the groove for the whole family of symmetric bulge profiles. For the typical groove widths and heights observed $\left(\sim 30^{\circ}\right.$ and $\sim 1.3$, respectively: see Paper II), the asymmetry should be just a few percent. Hence any larger north/south ratio would most probably be due to some physical reason and not to the effect of gas inflow geometry.

\subsubsection{Groove range vs. ionization bulge range}

The north and south ranges of the groove depend linearly on the range of the ionization rate profile. There is no dependence whatsoever of the range of the groove on the depth of the bulge, as shown in Fig. 6.

The position of minimum intensity (groove minimum) shows little dependence on the depth of the bulge (if at all, then for large widths), but it slowly moves towards southern latitudes with the increasing width of the bulge. The mean position of the groove, defined as the arithmetic mean of the north and south range, is practically independent of the ionization profile width. For the assumed latitude of the upwind direction, equal to $7.5^{\circ}$ north (equivalent to about $8^{\circ}$ heliolatitude), it goes level at $\sim 4^{\circ}$ south heliolatitude.

Hence, the observed position of the groove minimum should yield a clue on the symmetry of the north/south range of the ionization bulge. Another test would be to take the mean of the north and south groove range and see if they do not go too far away from the equator. Then, if these tests for symmetry of the ionization bulge are passed, one can infer the north and south range of the ionization bulge from the observed range (north and south) of the groove. With this information on hand, one can proceed to determine the height of the ionization bulge
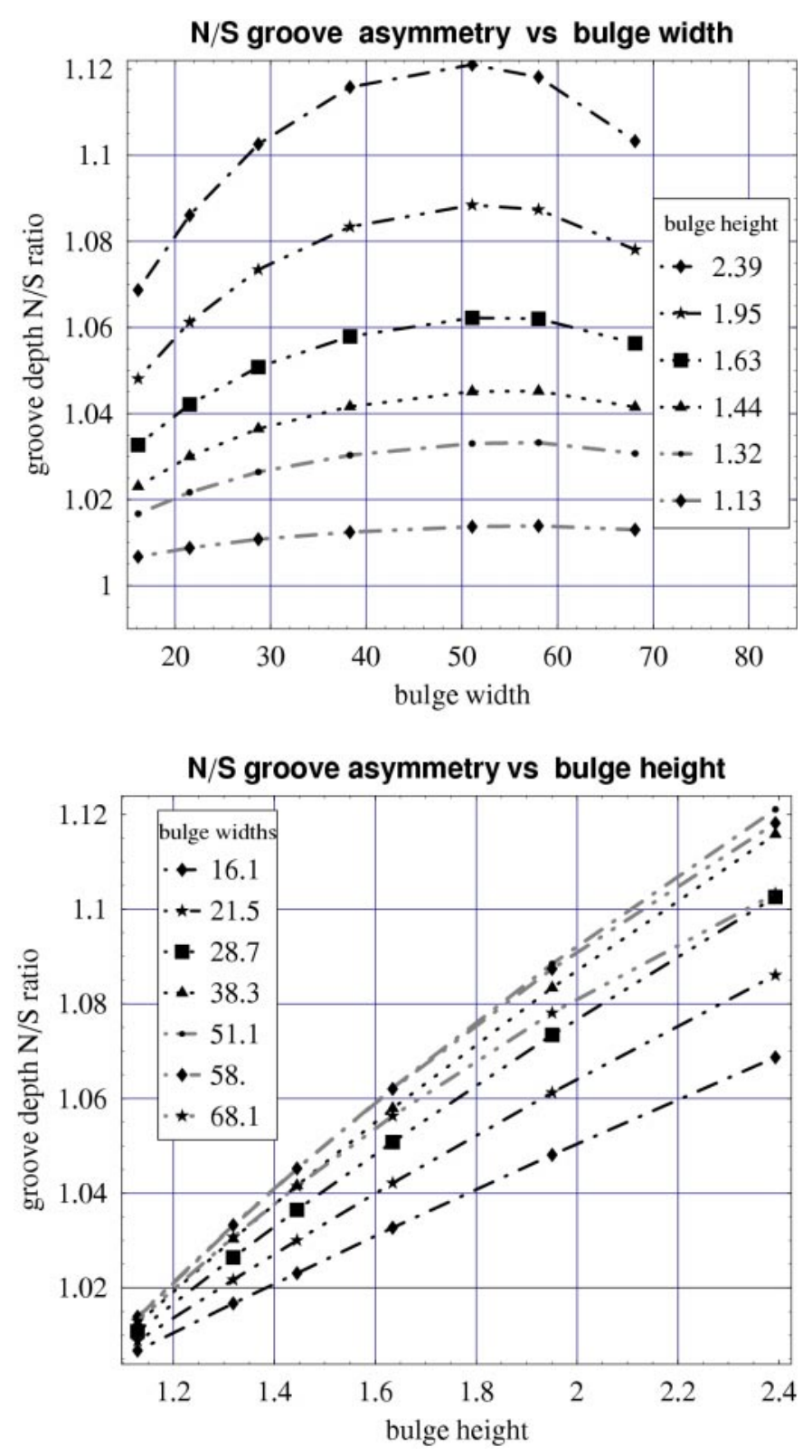

Fig. 5. Upper panel: N/S asymmetry of the groove depth as function of the bulge width for the bulge height values indicated in the box. For symmetric bulge, one does not expect more than $\sim 10 \%$ asymmetry. The highest occurs for the bulge width equal to about $50^{\circ}$. Lower panel: $\mathrm{N} / \mathrm{S}$ asymmetry of the groove depth as function of the ionization bulge height for bulge widths indicated in the box. The dependence is almost linear, but the slope varies with the width of the ionization bulge.

from the depth of the groove, which will be discussed in the next section.

\subsubsection{Groove depth vs. ionization bulge height}

The groove depth depends almost linearly on the bulge height, but some dependence exists also on the width of the bulge. Separate correlations occur for the north and south depths (see Fig. 7). The highest slopes occur for the highest widths of the bulge. The dependence of the groove depth on the 


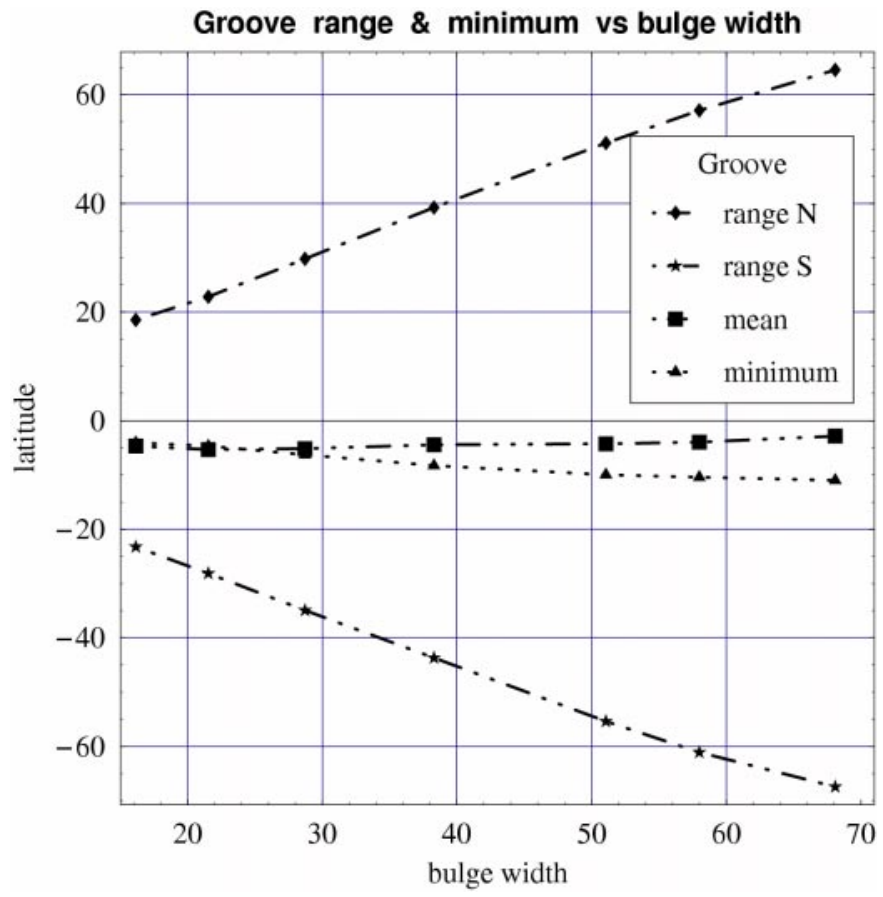

Fig. 6. Groove range north $(\mathrm{N})$ and south $(\mathrm{S})$ versus bulge range (here equivalent to the north and south width), as well as the latitude of the minimum of the groove and the arithmetic mean of its north and south range. The quantities shown are averaged over all bulge heights, but in the case of the north and south ranges the dispersion is smaller than the resolution of the figure. The dispersion of the two remaining parameters is just a few degrees.

ionization bulge width is not linear, though regular (see Fig. 8). The largest depths of the groove for a given height of the bulge occur for the bulge range equal to $\sim 30^{\circ} \mathrm{N}$ and $\sim 25^{\circ} \mathrm{S}$.

\subsection{Asymmetric bulge profiles}

The modelling in this case was performed only on a qualitative basis. The goal was to get some insight into the behavior of the groove profile when the ionization bulge is shifted to the south of the Sun, as suggested by some observations discussed in Paper II. I used the full range of the widths, but only one value of the depth, equal to 1.64. I checked the following two cases: one with the shift of the bulge to the south by $30^{\circ}$ (further on referred to as the "large shift"), and the other one with the shift by just $5^{\circ}$ (further on referred to as the "small shift"). The bulge profiles used in both cases are presented in Fig. 9 and the resulting groove profiles in Fig. 10.

Some of the ionization bulge profiles studied engulf at least partly the south pole. Hence the morphology of the resulting groove profiles can be quite different from the symmetric bulge case. The ionization bulge does not need to swallow the pole in order to obtain the groove profiles almost entirely flat in the southern hemisphere, but showing a considerable gradient in the northern hemisphere. This happens, e.g., for the groove profile corresponding to the bulge shifted to the south by $30^{\circ}$, width $38^{\circ}$ (cf. the lower panels of Figs. 9 and 10). Of course, when the bulge does engulf the south pole, that kind of groove
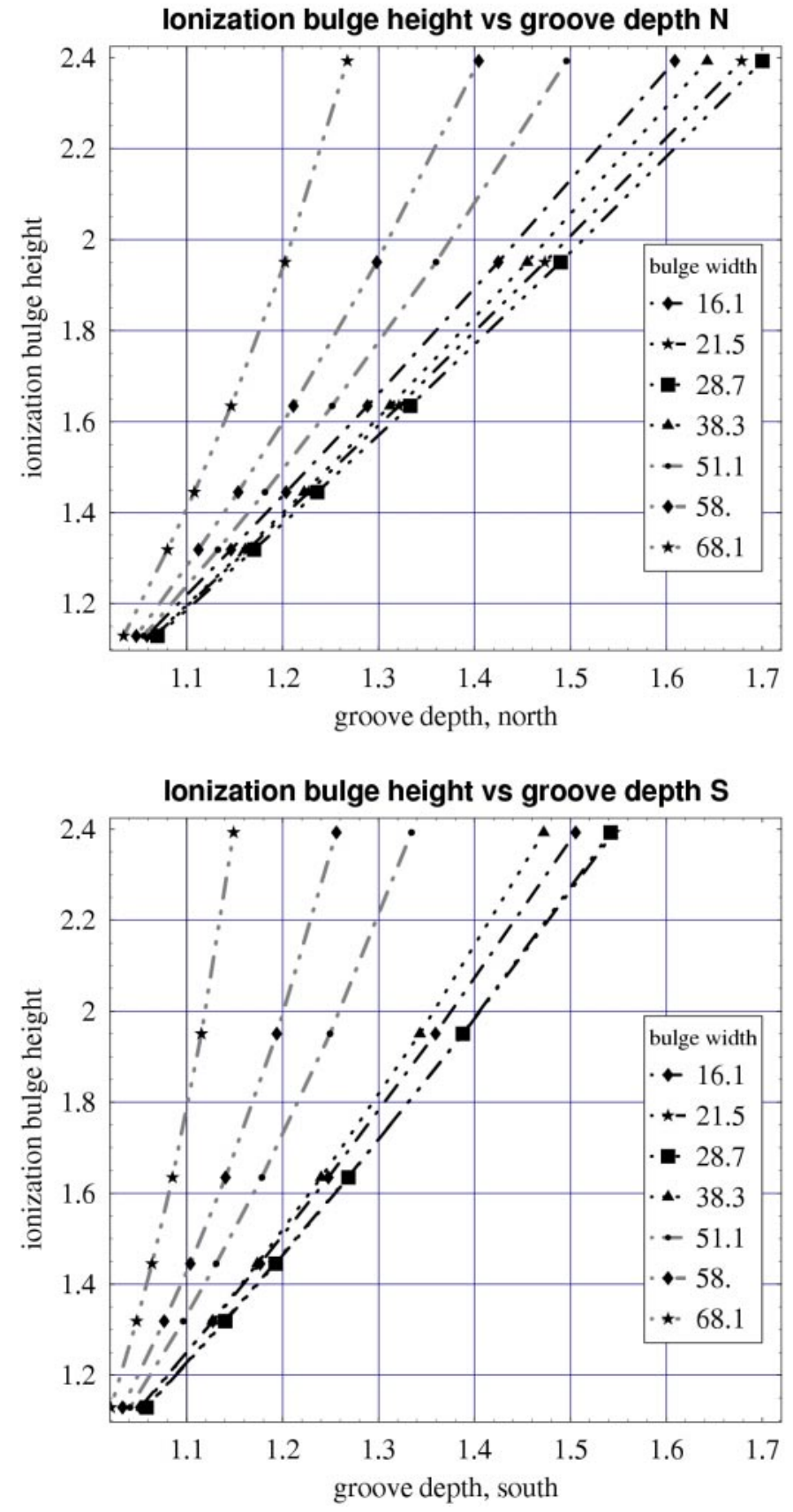

Fig. 7. Upper panel: height of the ionization bulge vs. depth of the intensity groove north for the bulge widths indicated in the box next to the drawing. For an individual width of the ionization bulge, the correlation between groove depth and bulge height is close to linear but the slope of the curve depends on the bulge width. Lower panel: as the upper panel but the quantities for the southern hemisphere are shown in the same scale - the slopes are somewhat different.

morphology occurs even in a greater extent (see the profiles for 51 through $68^{\circ}$ ).

Another striking change in comparison with the symmetric case is an increase of the north-south depth asymmetry, even when the shift is small. It is illustrated in Fig. 11. The difference in the north-south asymmetry is almost two-fold for typical depths of the bulge $(\sim 1.1$ compared with $\sim 1.06)$. In the 
Groove depth $\mathrm{S}$ vs bulge range $\mathrm{S}$

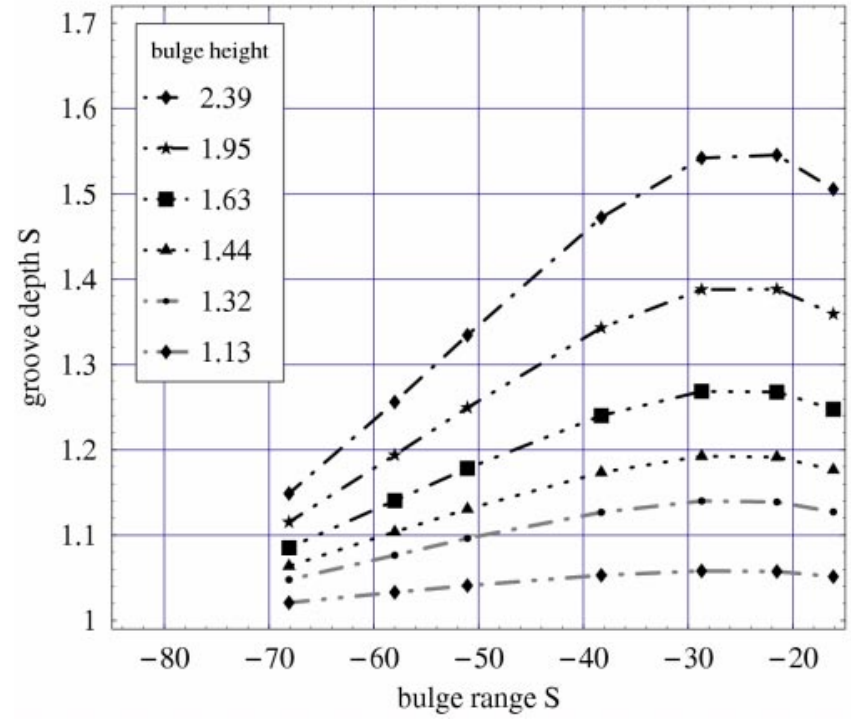

Groove depth $\mathrm{N}$ vs bulge range $\mathrm{N}$

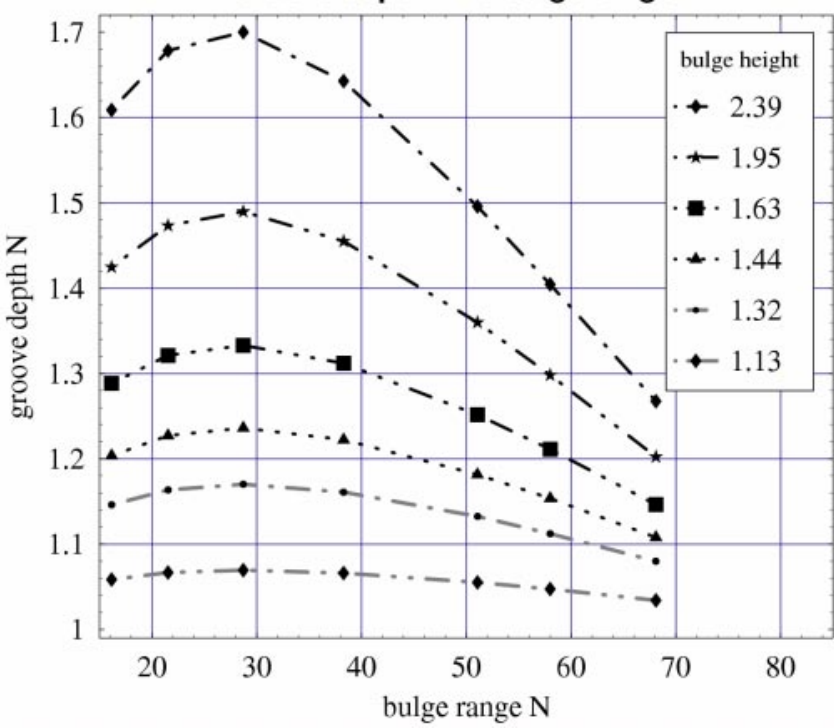

Fig. 8. Left-hand panel: groove depth north vs. bulge range north. Right-hand panel: groove depth south vs. bulge range south. Corresponding bulge heights are displayed in the boxes.

case of a big shift of the bulge the difference is immense but then one can see the shift at a glance.

Despite the large differences in the N/S asymmetry (see Fig. 12), the correlation between the range of the ionization rate profile and the range of the groove is conserved: in the model studied, the ionization rate profile is shifted by $5^{\circ}$ to the south and so is the groove. The same is valid even for the big shift of $30^{\circ}$. This means that despite possible shifts of the bulge with respect to the solar equator one is still able to read its north and south range from the range of the groove, at least as long as the groove does not engulf the south pole (notice the deviation from the straight line of the bulge range south, shown in Fig. 12, at about $75^{\circ}$ ).

A signature of the asymmetry of the range of the ionization bulge profile with respect to the solar equator may be the heliolatitude of the groove minimum (not to be confused with the mean value of the north and south range of the groove). A large shift is of course telltale of a shift of the bulge, but otherwise it is not clear without some analysis whether the bulge is symmetric about the solar equator or not. In the case of a small shift of the bulge, similarly as in the symmetric case, the heliolatitude of the minimum decreases to the southern hemisphere with the increase of the width of the ionization bulge, but in the case of the ionization bulge pushed slightly to the south the minimum of the groove profile is also pushed to the south. In the case studied the shift is 1.5 -fold higher than the shift in the symmetric case (see Fig. 13).

The correlation which existed between the groove range north and groove depth north in the symmetric case now is changed (see Fig. 14). This means that the groove profile is not suitable for determination of the actual height of the ionization rate profile from the extensive symmetric-case modelling we performed.

\subsection{Procedure to determine parameters of the ionization bulge from the observed parameters of the intensity groove}

The procedure of determining of the ranges and heights of the ionization bulge will be explained on example. Let us assume that an observed groove profile passed the tests for symmetry of the underlying ionization bulge profile, discussed in the preceding subsection, and that from the northern limb of the groove profile we measured the range equal to $40^{\circ}$ and depth equal to 1.3. The procedure to infer the bulge parameters will be the following. From the linear relation between the range of the bulge and the range of the groove (Fig. 6) we immediately read off the bulge range equal to $39.1^{\circ}$ and then, using the relations presented in Fig. 7, we tabulate the bulge heights as function of the bulge range for the groove depth observed (Fig. 15). From this table we calculate by interpolation that the bulge height is equal to 1.62 .

The southern limb of the groove is processed in a similar way to establish the parameters of the southern limb of the ionization bulge.

The method presented is simple and lacks sophistication needed to interpret more complicated groove profiles, observed by SWAN during solar maximum. It is useful for the cases when the groove is relatively narrow and close to the solar equator. Its application to actual observations brought the results presented in Paper II.

\section{Discussion}

The geometry of observations adopted in Paper II (all lines of sight perpendicular to the inflow direction and either in or close to the upwind hemisphere, the observations point very close to the upwind or downwind axis at $1 \mathrm{AU}$ ) and the use of relative 

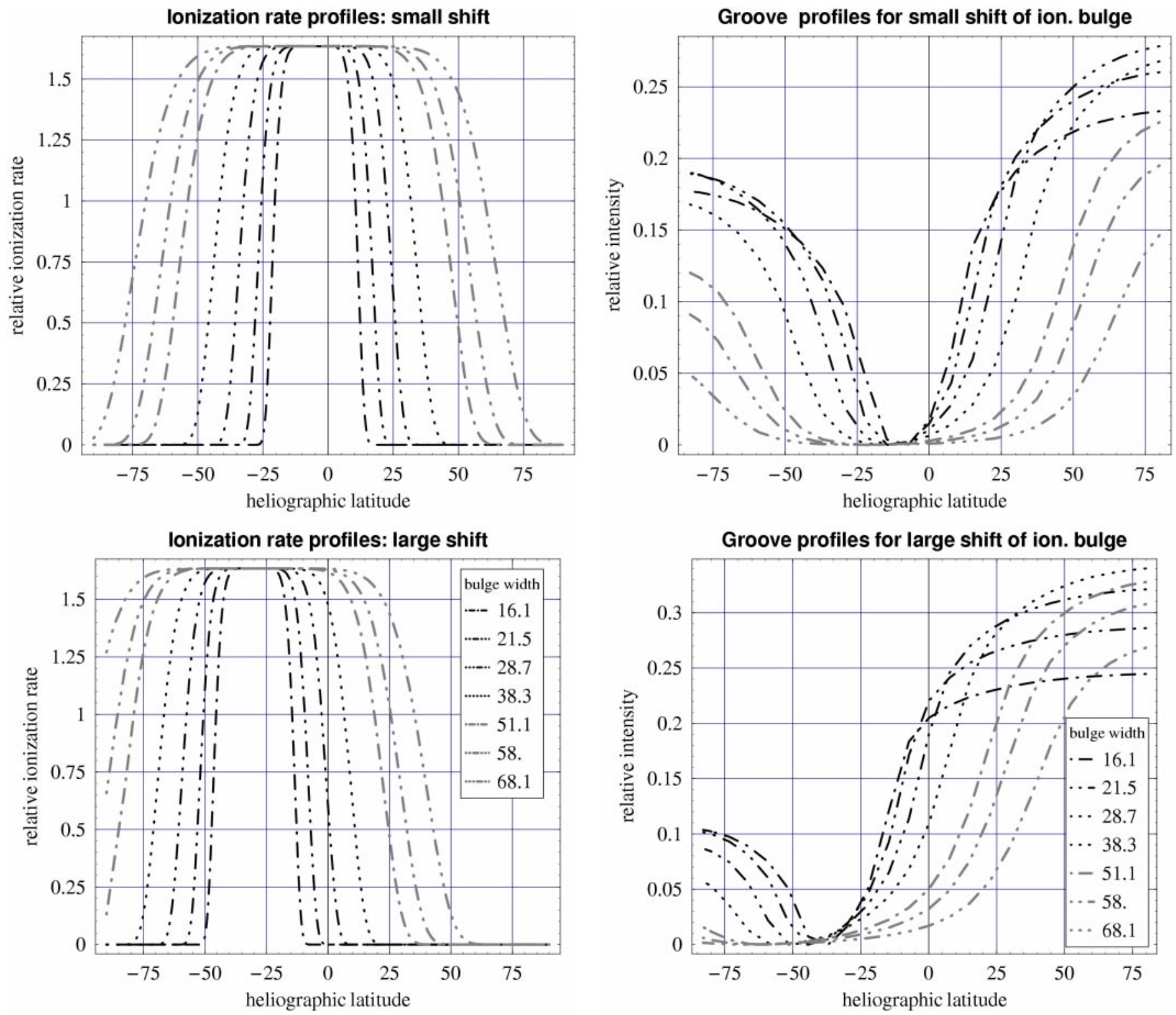

Fig. 9. Upper panel: family of ionization bulge profiles shifted by $5^{\circ}$ to the south with respect to the solar equator. Lower panel: family of ionization bulge profiles shifted by $30^{\circ}$ to the south with respect to the solar equator. The widths of the profiles are indicated in the box. Note that in this geometry, beginning from the width equal to $\sim 50^{\circ}$ the ionization bulge starts to engulf the south pole.

profiles instead of absolute intensities eliminate or strongly reduce most of the unwanted factors that could affect the results. These factors include:

- multiple scattering of solar Lyman- $\alpha$ photons on the heliospheric gas;

- axially symmetric distribution of interstellar hydrogen at the termination shock of the solar wind, in contrast to spherical symmetry assumed in the classical models;

- uncertainty of absolute density of the gas at the termination shock;

- uncertainty of the bulk velocity and temperature of the gas at the termination shock;

Fig. 10. Heliospheric backscatter groove profiles for the bulge profiles as shown in Fig. 9. Upper panel: for the small shift to the south, lower panel: for the large shift to the south. The bulge widths, identical for the two shifts, are indicated in the box.

- uncertainty of the absolute values of the radiation pressure and its variation in time;

- uncertainty of the net value of the ionization rate at solar equator and of its variations in time;

- difference between the geometry of modelling and the geometry of actual observations.

The multiple scattering does not affect the results since, as shown by Quémerais (2000) and Quémerais \& Izmodenov (2002), it introduces a bias by a factor that depends strongly enough on the offset angle from the upwind direction, but only mildly on the parameters of the gas at the termination shock. For the offset angles $\theta$ less than $\sim 135^{\circ}$, that is also for our lines of sight, the correction is only a few percent. Since we use relative intensities constructed from lines of sight with 


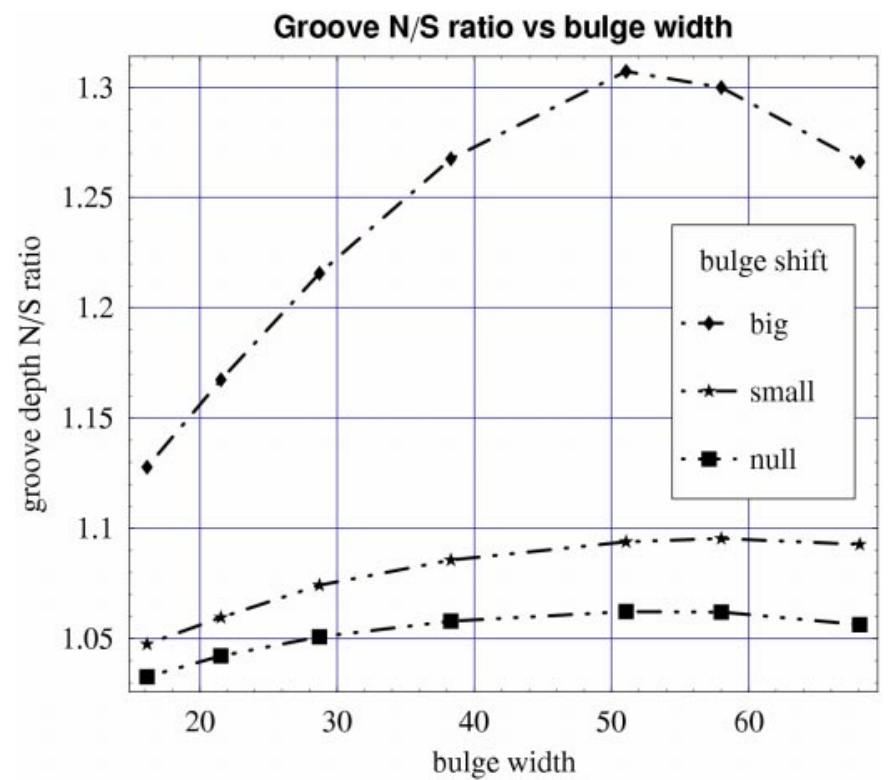

Fig. 11. N/S asymmetry of the groove depths as function of the ionization bulge width for the big, small and null shift of the ionization bulge to the south.

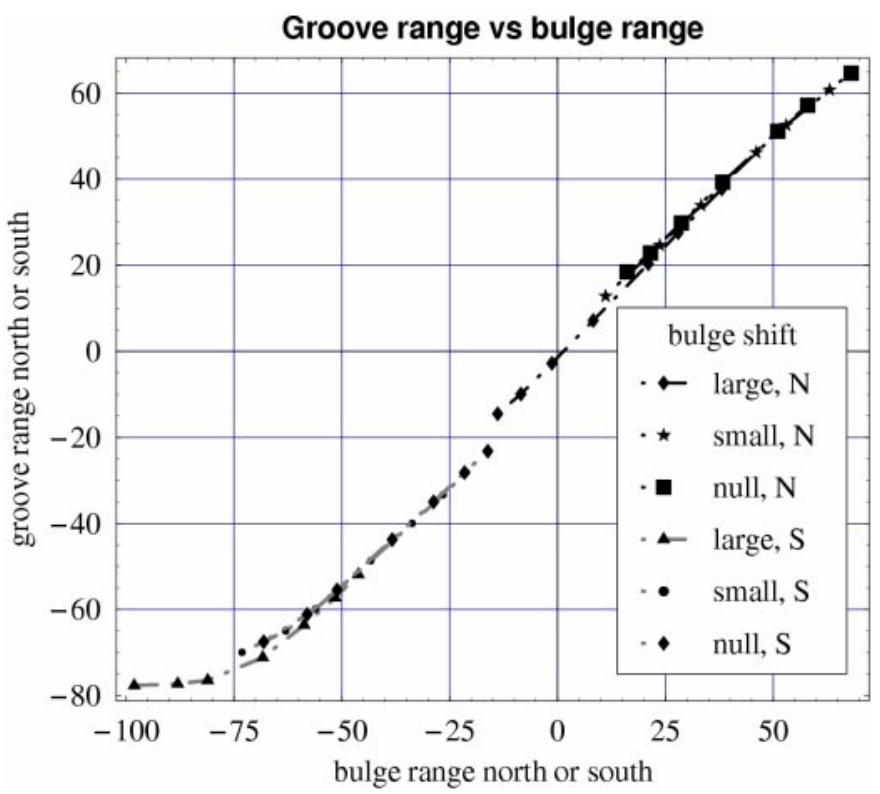

Fig. 12. Groove range to the north $(\mathrm{N})$ and to the south $(\mathrm{S})$ as function of the north and south range of the ionization bulge for the big, small and null shift of the bulge profile to the south. The cases of small and null shift are almost identical.

common $\theta$ angle, the small multiplicative corrections cancel each other out.

The heliospheric interface in the absence of magnetic field is an axially symmetric structure, with the axis of symmetry coincident with the upwind axis (Baranov et al. 1991; Osterbart \& Fahr 1992; Zank et al. 1996). Hence it does not change relations between densities of the hydrogen gas in the planes perpendicular to the inflow direction, though it does change the relations between the gas densities in other planes (Quémerais \& Izmodenov 2002). Therefore the influence of the interface

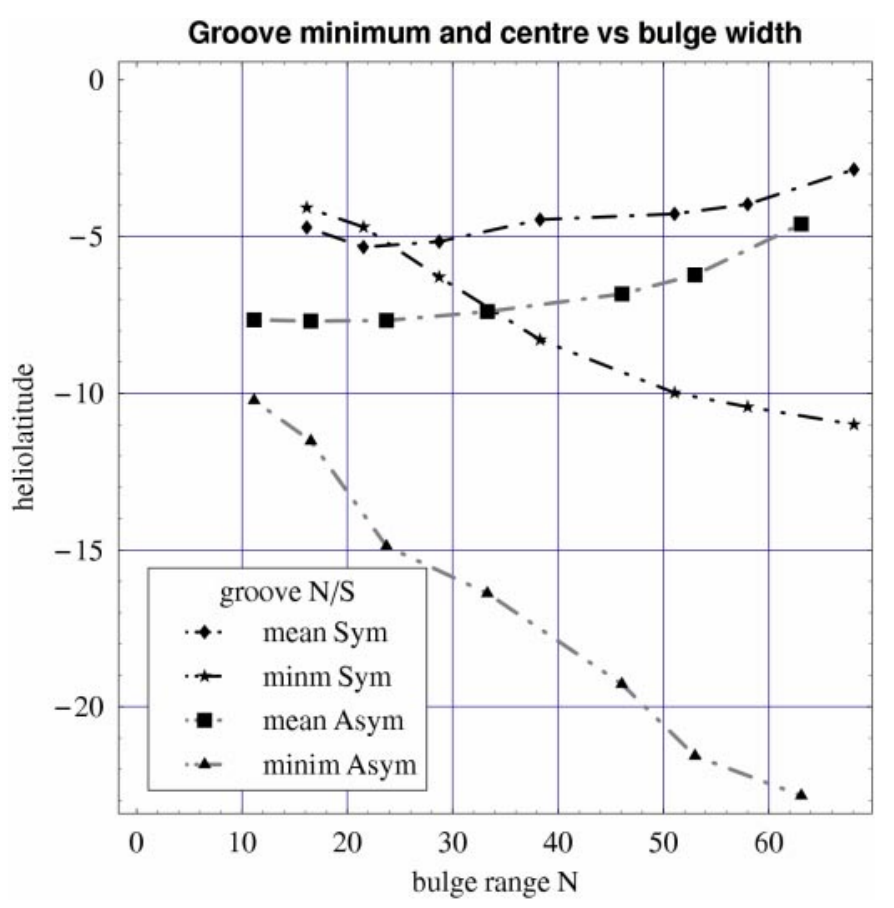

Fig. 13. Heliographic latitude of the minimum of the groove profile ("minim" in the box) and of the arithmetic mean of the groove range north and south (marked "mean" in the box) for the case of small and null shift of the ionization bulge to the south (marked "asym" and "sym" in the box).

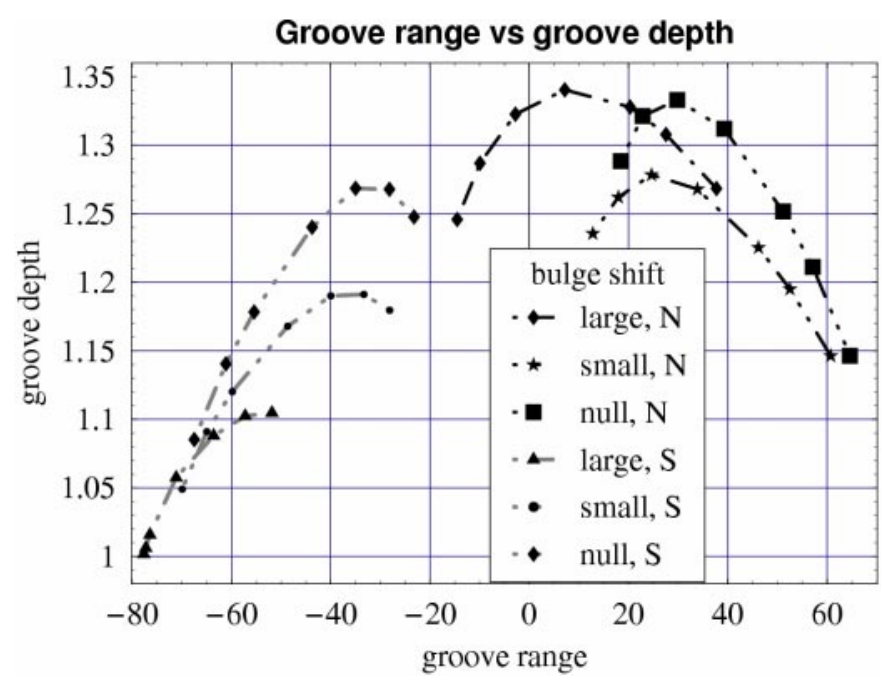

Fig. 14. Groove depth north and south as function of the groove range north and south for the big, small, and null shift of the ionization bulge. The correlations exist but they are quite different for each of the shifts, which prevents one from using them to determine the ionization bulge height from observed depth of the groove if evidence exists that the ionization bulge is not symmetric about the solar equator.

on the local gas distribution is not a factor in this study. One must keep in mind, however, that the interface modelling performed so far has been done under assumption that the solar wind is spherically symmetric. An exception is a study by Pauls \& Zank (1996), but their results are not directly applicable to the region observed by Lyman- $\alpha$ photometers located at $1 \mathrm{AU}$, because these instruments are not able to see the heliospheric 
Bulge height vs bulge range for groove depth 1.3

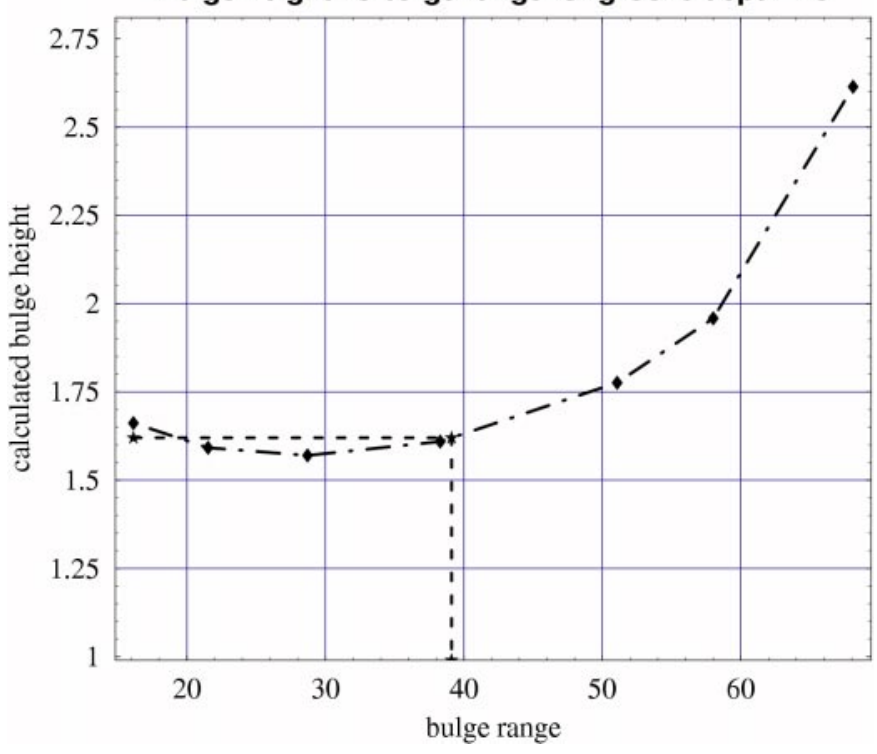

Fig. 15. llustration of determination of the ionization profile height from groove observations. The bulge range - bulge height relation is tabulated as function of the groove range for the depth of the groove observed. Then the bulge height value is calculated by interpolation for the bulge range inferred from the groove range (Fig. 6). The numerical values of the example groove parameters are north range $40^{\circ}$ and north depth 1.30 .

Lyman- $\alpha$ glow farther than $\sim 20$ AU in the crosswind plane. A departure from axial symmetry can be, a priori, expected in the presence of an arbitrarily directed extraheliospheric magnetic field. No quantitative analysis of behavior of neutral hydrogen in such conditions is available, though, and Quémerais et al. (1999) showed that the direction of flow of the heliospheric hydrogen observed by SWAN is deflected at most by just a few degrees from the upwind direction determined for the interstellar helium by Witte et al. (1996) and Lallement \& Bertin (1992). Therefore I really do not expect any important error from this source in the analysis.

Since I use the optically thin, single scattering model, the absolute intensity scales linearly with the absolute density of the gas at the termination shock, and since I analyze relative intensity profiles, the absolute intensity is eliminated. Hence also eliminated is the influence of uncertainty of the absolute calibration of the instrument.

The absolute intensity profiles show some dependence on the bulk velocity and temperature of the gas at the termination shock. But I analyze relative intensity profiles in the crosswind plane, that is for constant offset angles $\theta$. As demonstrated by Bzowski et al. (2002), such profiles show very little dependence on these parameters. Further, I performed the calculations for the bulk speed and temperature close to the currently best estimate of these parameters (Costa et al. 1999; Lallement 1999), so the margin for error is even more reduced.

Time dependent effects due to solar-cycle variations of the solar radiation pressure and ionization rate, studied in the context of this research by Bzowski et al. (2002), do not play a significant role because we restrict the lines of sight to the crosswind plane. This would not be the case in any other plane.
This concession was verified by support modelling presented in Paper II, where the results obtained on the basis of the theory presented here were satisfactorily reproduced with the use of the full time- and latitude-dependent model of the gas distribution.

\section{Conclusion}

From the modelling discussed above I infer the existence of specific correlations between the height and range of the ionization bulge on one hand and the depth and north and south range of the groove in the heliospheric Lyman- $\alpha$ glow on the other hand. Based on these correlations and keeping in mind the observations of the groove presented in Paper II, the following suggestions for the interpretation of the actual groove profiles are put forward.

1. Observe the profiles of intensity of the heliospheric Lyman$\alpha$ glow from $1 \mathrm{AU}$ upwind and downwind from the Sun, making $360^{\circ}$ scans in the plane perpendicular to the gas inflow axis. Render the results as function of the heliographic latitude.

2. Find the position of the groove minimum and its north- and south depths. If the depths are large (1.2-1.3) and the minimum position is about $5^{\circ} \mathrm{S}$, then most probably the ionization rate profile is symmetric about the solar equator. Based on the modelling discussed, it will be possible to determine the range and height of the ionization rate profile from the north and south depths and ranges of the groove profile. If the ratio of intensity at the north solar pole to the intensity at the south solar pole exceeds $\sim 1.1$, then surely a largescale north/south asymmetry of the ionization field is expected. Further, if an independent information on the photoionization rate of heliospheric hydrogen is available, one can draw conclusions about possible north-south asymmetries of the intensities of the fast solar wind emanating from the polar holes.

3. If the latitude of the minimum is a little bit farther south than the $\sim 5^{\circ}$ and the depth is still large, then most probably the ionization rate profile is slightly pushed to the south. It will be possible to determine the north and south ranges of the ionization bulge with a little bit larger error than in the former case. Determination of the depths, however, will not be credible and hence discussion of the north-south asymmetries of the flux of the solar wind from the polar holes will not be possible. If the depth of the groove is small, then a moderate southern shift of the groove minimum need not be related to the shift of the ionization bulge but it rather occurs as a consequence of the increased latitudinal range of the bulge.

4. If the latitude of the groove minimum is large (it occurs at southern mid-latitudes) and the depth is smaller than in the former cases, then most probably the ionization rate profile (the ionization bulge) is considerably pushed to the south. In this case, if the groove profile is regular enough, it is still possible to determine the north and south range of the ionization bulge, though the southern one with reduced accuracy. But the correlations between the groove depth and 
range both north and south are different than in the symmetric case, so concluding on the height of the ionization rate profile will not be possible. Any conclusions will be more of qualitative than quantitative nature.

Acknowledgements. I gratefully acknowledge discussions with Daniel Ruciński, Tuula Summanen, Erkki Kyrölä, and Rosine Lallement. I thank Erkki for reading the manuscript. This research was supported by the Polish State Committee for Scientific Research Grant 2P03C 00519.

\section{References}

Baranov, V. B., Lebedev, M. G., \& Malama, Y. G. 1991, ApJ, 375, 347 Bzowski, M. 2001a, Space Sci. Rev., 97, 379

Bzowski, M. 2001b, in The Outer Heliosphere: The Next Frontiers, ed. K. Scherer, H. Fichtner, H. J. Fahr, \& E. Marsch, COSPAR Coll. Ser. 11 (Pergamon: Elsevier), 69

Bzowski, M., Summanen, T., Ruciński, D., \& Kyrölä, E. 2002, J. Geophys. Res., 107, 10.1029/2001JA00141

Costa, J., Lallement, R., Quémerais, E., et al. 1999, A\&A, 349, 660

Fahr, H. J. 1978, A\&A, 66, 103

Izmodenov, V. V., Gruntman, M. A., \& Malama, Y. G. 2001, J. Geophys. Res., 106, 10681
Lallement, R. 1999, in Solar Wind Nine, AIP Conf. Proc., 471, 205 (American Institute of Physics)

Lallement, R., Bertaux, J. L., \& Kurt, V. G. 1985, J. Geophys. Res., 90, 1413

Lallement, R., \& Bertin, P. 1992, A\&A, 266, 479

Osterbart, R., \& Fahr, H. J. 1992, A\&A, 264, 260

Pauls, H. L., \& Zank, G. P. 1996, J. Geophys. Res., 101, 17081

Phillips, J. L., Bame, S. J., Barnes, A., et al. 1995, Geophys. Res. Lett., 22, 3301

Quémerais, E. 2000, A\&A, 480, 353

Quémerais, E., Bertaux, J.-L., Lallement, R., et al. 1999, J. Geophys. Res., 104, 12585

Quémerais, E., \& Izmodenov, V. V. 2002, A\&A, 396, 269

Ruciński, D., \& Bzowski, M. 1995, A\&A, 296, 248

Ruciński, D., \& Bzowski, M. 1996, Space Sci. Rev., 78, 248

Thomas, G. E. 1978, Ann. Rev. Earth Planet. Sci., 6, 173

Tobiska, W. K., Pryor, W. R., \& Ajello, J. M. 1997, Geophys. Res. Lett., 24, 1123

Witte, M., Rosenbauer, H., Banaszkiewicz, M., \& Fahr, H. J. 1996, Space Sci. Rev., 78, 289

Wu, F. M., \& Judge, D. L. 1979, ApJ, 231, 594

Zank, G. P., Pauls, H. L., Williams, L. L., \& Hall, D. 1996, J. Geophys. Res., 101, 21636 